
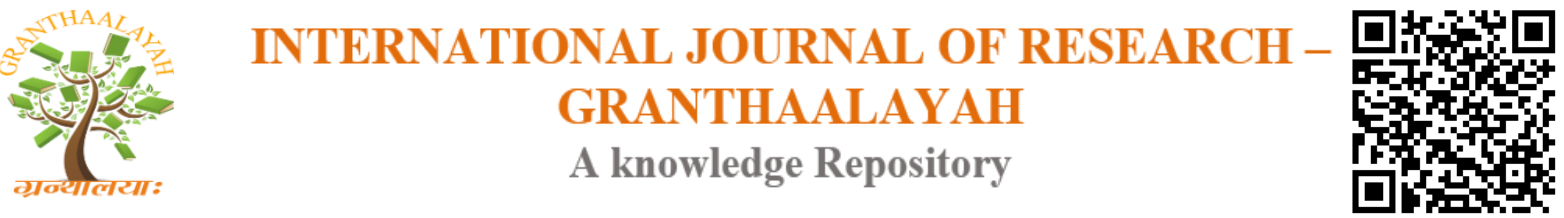

Science

\title{
BUTTER CAKE ENHANCED WITH MALUNGGAY (MORINGA) POWDER AT CEBU TECHNOLOGICAL UNIVERSITY, MAIN CAMPUS: TECHNO GUIDE
}

\author{
Vivian B. Delos Reyes ${ }^{1}$, Adora A. Villaganas ${ }^{2}$, Victor D. Villaganas ${ }^{3}$ \\ ${ }^{1}$ Professor, College of Education, Cebu Technological University, Cebu City, Philippines, 6000 \\ ${ }^{2,3}$ Professor, Graduate School, Cebu Technological University, Cebu City, Philippines, 6000
}

\begin{abstract}
This study was conducted to formulate a butter cake enhanced with moringa powder in four (4) different levels for three replications. Experimental method of research with researcher made score sheet was utilized. The sensory evaluation used by the researcher made score sheet designed for the nine point-hedonic rating scale tests and the data were treated with arithmetic mean to determine the most acceptable treatment. The data were further statistically treated using the Analysis of Variance (one and two-way) to get the significant mean difference between the treatments. The most acceptable treatment was subjected for proximate and Vitamin A analyses to determine the nutritive values of the product. In this endeavor economic value was also computed to determine the economic feasibility for production. The findings revealed that treatments one (1) and two (2) containing smaller amount of moringa powder came out the most acceptable formulations having a verbal description of like very much. Between these two treatments, treatment two earned higher numerical mean rating of 7.26/7.92 by the professors and students/panelists respectively and perceived the color as medium low olive green, with moderately pleasant taste of moringa, moderately desirable flavor, and texture of slightly less moist and tender got the highest overall sensory acceptability of the product. Aside from this other vital nutrients found in the product is Vitamin A which is recorded 450 International Unit (IU) per slice $/ 100 \mathrm{~g}$ amazingly surpasses the contents of the standard recipe. This is due to moringa powder addition. The return of investment of Php.34 per every peso is likewise found to be a very good business to start.
\end{abstract}

Keywords: Butter Cake; Moringa Powder; Cebu Technological University.

Cite This Article: Vivian B. Delos Reyes, Adora A. Villaganas, and Victor D. Villaganas. (2017). "BUTTER CAKE ENHANCED WITH MALUNGGAY (MORINGA) POWDER AT CEBU TECHNOLOGICAL UNIVERSITY, MAIN CAMPUS: TECHNO GUIDE." International Journal of Research - Granthaalayah, 5(7), 14-25. https://doi.org/10.29121/granthaalayah.v5.i7.2017.2104. 


\section{Introduction}

Malunggay contains high in Vitamin A, iron, calcium, potassium and many other nutrients. Vitamin A is fat soluble, therefore if it is not prepared accordingly the vitamin A is not totally utilized by the body. So, the nutrients then are just wasted. Utilization of malunggay other than the usual way of cooking as part of the viand is the focus of this study. One way of introducing malunggay to the children and malunggay biased people is integrating malunggay into butter cake. Thus, this study was conducted at aiming and investigating the acceptability of butter cake enhanced with malunggay. Acceptability doesn't end at the testing phase only but also includes the nutritional aspect of the product. People nowadays are health conscious.

Food is one of the basic needs of man that has to be met accordingly. Daily meal planning must be adequate from the nutritional standpoint considering the dinners. Report on health status of Filipinos improved in terms of the decrease in the mortality rates of mothers and infants, the rising incidence of infectious diseases such as diarrhea and respiratory diseases contributed to the poor nutritional status of many Filipinos. This explicitly advocates the utilization and implementation of proper nutrition and well balances meals in every family. Today's life style changes the ways of preparing food, cooking, serving and eating. The demands of the time, preferences for ready to eat or instant foods, availability of different food chains and many more alternative choices without so much concern on the nutritional values are just among the many reasons for this cause. In the abstract cited in the Philippines poverty and pervasive malnutrition, it was pointed out that these concerns are not only limited to families of deprived seasonal workers. Undernourishment is endemic and increasing throughout most of the archipelago of some 7100 islands. Ravenholt A. (Abstract FAO Regional Site for Asia \& South East Pacific Philippines Google) date visited July2, 2010.

\subsection{Related Literatures}

Gan in his book, Your Health and Mind stressed the findings that the deficiencies in food intake among Filipinos in general are due to a number of reasons among which are: (1) lack of knowledge of what is essential to a satisfying diet, (2) failure to make efforts to attain adequate diet, (3) failure of people to apply what little nutrition knowledge they have acquired, (4) undeveloped tastes for the more essential foods and finally, (5) unintelligent health behavior in which one does what he wants and eats he please [1]. This study relates to the present in terms of vegetable utilization for nutritional value.

Minor changes in eating habits can lead to major changes in health. What is eaten not only affects the day-to-day health but also helps to determine the quality and length of life.

Cakes of different kinds are well loved by Filipino people. The growth of small bakeries and large bakeshops that offer simple to special cakes indicate that cakes have become part of Filipino meals. Many people have gone into retail selling of cakes or supplying cakes in groceries, bakeshops, offices, canteens, food convenience stores and stalls. People discovered various ways to create demand for cakes nowadays such as personal selling, taste sampling, sales promotion, and resort to advertising for larger business or selling cakes at discounted prices for 
introductory selling. [2] stated that product innovation is one of the key factors in food production.

Innovation is the key to profitability in competitive world market. It is essential that people become better at food production and food processing through innovation if one is to compete on a level playing field with other large producing nations through optimum utilization of indigenous resources in the locality for example the malunggay (Moringa). Cake baking is a good business but it entails a lot of hardships before success can be achieved. In the end, perseverance and dedication makes a difference. Cake is a form of food, typically a sweet, baked dessert. Cakes normally contain a combination of flour, sugar, eggs, and butter or oil, with some varieties also requiring liquid (typically milk or water) and leavening agents (such as yeast, baking powder and baking soda). Flavorful ingredients like fruit purées, nuts or extracts are often added, and numerous substitutions for the primary ingredients are possible. Cakes are often filled with fruit preserves or dessert sauces (like pastry cream), iced with butter cream or other icings, and decorated with marzipan, piped borders or candied fruit. Cake is often the dessert of choice for meals at ceremonial occasions, particularly weddings, anniversaries, and birthdays.

There are countless cake recipes; some are bread-like, some rich and elaborate and many are centuries old. Cake making is no longer a complicated procedure; while at one time considerably laborious to go into cake making (particularly the whisking of egg foams), baking equipment and directions have been simplified that even the most amateur cook can bake a cake. In cake making, bakers need to know the basic ingredients needed as well their economic and nutritive value. The cost of ingredients used in cake making is one factor that affects the profitability of the baked products. In the country side, homemakers must be challenged to produce quality products using ingredients found within the locality. Bakers for example, can switch to the use of some ingredients they can locally produce rather than buy those ready in the market which demand higher prices. [3]. Cakes can be generally classified according to the formula or ingredients used.

\section{Materials and Methods}

\subsection{Methodology}

The study availed with the experimental method. The treatment formulations used were based on the basic ingredients and baking procedures of butter cake. Find out how butter cake basic recipe can be enhanced there are four (4) levels of moringa powder added to the basic recipe. Treatment 1 one (1) tablespoon of moringa powder, Treatment 2 two and one-haft (2.5) tablespoons, Treatment 3 three and a-haft (3.5) tablespoons, and Treatment 4 four and a-haft (4.5) of moringa powder added. Since the study aimed to formulate an acceptable butter cake enhanced with moringa powder sensory attributes were observed: color, taste, flavor and texture of the finished product. Samples were labeled prior to sensory analysis using 4-digit code numbers reflected in the score sheet correspondingly to the code numbers found in the serving tray. The four digit code was designed to avoid biases or product formulation identification by the panelists. The nine-point hedonic rating scale which were reflected also on the score sheet were tallied and computed to determine the mean average per product sensory attribute in the 
application. The data gathered were subjected to further statistical analysis using the ANOVA (Analysis of Variance) for comparison and interpretation to determine the significant mean difference among the four (4) treatment formulations. The output is a techno guide of BSED-BT students.

\subsection{Research Instrument}

The instrument used was a self-made by the researcher. A dry run was conducted prior to the final sensory evaluation. An orientation on the use of the score sheets was made so that the panelists were fully understood the meaning of the qualitative interpretation of the hedonic-rating scale as reflected in the instrument.

\subsection{Statistical Instrument}

The data gathered from the sensory evaluation both from the experts and students were treated statistically employing the following formula:

1) The Arithmetic Mean;

2) Average Weighted Mean;

3) Analysis of Variance (ANOVA); and

Arithmetic Mean- This technique was used to measure the central tendency. This was used to gauge the average value of responses to items on the questionnaire. The formula was applied in all of the three replications.

The formula is: $\quad X=\sum_{n} X$

Where: X - Arithmetic Mean

$$
\begin{aligned}
& \sum \text { - Summation Symbol } \\
& \mathrm{X} \text { - Scores } \\
& \mathrm{n} \text { - Number of Respondents }
\end{aligned}
$$

Average Weighted Mean. Average is used to determine the mean scores.

The formula is: $\mathrm{AWM}=X=\sum_{n} X$

$$
\begin{aligned}
& \text { Where: } \sum \text { - Summation Symbol } \\
& \mathrm{X}-\text { Arithmetic Mean } \\
& \mathrm{n}-\text { Number of Cases }
\end{aligned}
$$

Analysis of Variance (ANOVA)- The Analysis of Variance (ANOVA) was computed to determine the significant mean difference between treatments. The following statistical formulas will be followed. The following statistical treatments of contributing factors were subjected to FTest at $5 \%$ and $1 \%$ level of significance.

One-way analysis of variance (ANOVA) tests measure significant effects of one factor only, two-way analysis of variance (ANOVA) tests (also called two-factor analysis of variance) measure the effects of two factors simultaneously. 
Two-way ANOVA on the other hand would not only be able to assess both time and treatment in the same test, but also whether there is an interaction between the parameters. A two-way test generates three p-values, one for each parameter independently, and one measuring the interaction between the two parameters.

From the data gathered in the sensory acceptability arithmetic mean, Analysis of Variance (ANOVA) was applied using the following formula:

$\mathrm{F}=$ Mean Square Treatment Mean Square Error

\section{Results and Discussion}

The analysis and interpretation of data gathered is based on the acceptability of the butter cake enhanced with moringa powder.

\subsection{Findings}

Based on the results of the data gathered from the butter cake enhanced with moringa powder uncovered the following findings:

\subsection{Different Levels Of Butter Cake Enhanced with Moringa Powder}

Considering the general sensory attributes such color, taste, flavor and texture for the four butter cake products the panelists revealed that:

Treatment one $\left(\mathbf{T}_{\mathbf{1}}\right)$ - One (1) tablespoon of moringa powder added. Color acceptability was low light olive green. Both panelists described the product as low light olive green. The taste as to professor panelists test recorded a pleasant moringa but the student panelists perceived it with moderately pleasant. Both panelists found the flavor moderately desirable. Texture, both groups of evaluators perceived the product slightly less moist and tender and with the total weight is 678 grams with thickness is $7 / 8 \mathrm{~cm}$.

Treatment two $\left(\mathbf{T}_{2}\right)$ - Both panelists perceived the product with medium low olive green color. The moringa taste is moderately pleasant, the flavor is found moderately desirable, the texture is slightly less moist and tender, total weight of 680 grams and the thickness is $7 / 8 \mathrm{~cm}$.

Treatment three $\left(\mathbf{T}_{\mathbf{3}}\right)$ - Both group of panelists described as color medium dark olive green, the moringa taste is very pleasant, the flavor is slightly desirable, the texture is slightly less moist and tender; the total weight is 683 grams and the thickness is the same $15 / 16 \mathrm{~cm}$.

Treatment four $\left(\mathbf{T}_{\mathbf{4}}\right)$ - Described as dark olive green, the moringa taste is very pleasant, as to flavor professor panelists found product as slightly desirable but the student panelists found it moderately desirable, professors panelists perceived texture as slightly dry and rubbery while the student panelists see it as slightly less moist and tender, the total weight is 688 grams and the thickness is 1 inch. 
It can be noted that all treatments has a very slight difference in thickness. In terms of color shades of green differ due to the amount of moringa added on the mixture. The pleasant of moringa also differs as the amount of moringa powder is increased. The more moringa addition to the mixture the darker is its color and the more pleasant of moringa is observed which the evaluators seemingly dislike. The lesser the amount of moringa added to the mixture the higher were the ratings given by the evaluators.

\subsection{General Acceptability of The Butter Cake Enhanced with Moringa Powder}

Based on the general sensory attributes for the product's acceptability, all the four sensory characteristics like color, taste, flavor and texture were given emphasis in this analysis.

Treatment one $\left(\mathbf{T}_{\mathbf{1}}\right)$ - One (1) tablespoon of moringa powder added. Color acceptability was low light olive green; taste recorded moderately pleasant, flavor revealed moderately desirable, and texture slightly less moist and tender with a verbal description of like very much $(7.25 / 7.89)$ rating in overall sensory acceptability of the product to both group of panelist.

Treatment two $\left(\mathbf{T}_{2}\right)$ - This treatment contained two and haft (2.5) tablespoons of moringa powder which is perceived as medium low olive green, with a taste of moderately pleasant, moderately desirable flavor, and texture of slightly less moist and tender with a verbal description of like very much (7.26/7.92) in the overall acceptability on the characteristics quality of the product for both panelist of evaluators.

Treatment three $\left(\mathbf{T}_{\mathbf{3}}\right)$ - Three and haft (3.5) tablespoons of moringa powder were added to this treatment that obtained a color description as medium dark olive green, with very pleasant moringa taste, slightly desirable flavor, and with a texture of slightly less moist and tender which obtained the different verbal description from each group of panelist. The professors group of evaluators recorded a verbal description of like moderately (7.05) while the students group revealed a verbal description of like very much (7.56). Professor panelists are more sensitive to the sensory quality that may have affected their level of acceptability to the product while the student panelists who happen to be on the adolescents' age group are gourmet by the nature of their age and not very sensitive to specific quality characteristics of food, to them what is important is that the food taken in can answer their hunger.

Treatment four $\left(\mathbf{T}_{4}\right)$ - Four and haft (4.5) tablespoons of moringa powder were added. A product characterized with dark olive green color, having a very pleasant moringa taste, with a flavor that is slightly desirable, and texture slightly less moist and tender. The professors' evaluators rated this treatment with a verbal description of like slightly (5.71) while the students' evaluators gave a verbal rating of like moderately (7.22) in the overall acceptability level of the product. Again the two groups of panelists seemed to have different sensory acceptability level on this treatment. Professor panelists are more keen and observant as they evaluate the product while the students group with adolescents' age bracket is less sensitive but foodie. However, it can be noted that from treatment three to four in both group their acceptability level range were descending. Considering the most acceptable among the four treatments, it is revealed that treatment two occupy the highest slot with a very slight numerical difference with treatment one. Although these two treatments fall on the same verbal description, like very much. The material cost in all 
treatments is the same, the researcher preferred treatment two for the proximate analysis due to nutritional value that may have been added from moringa powder.

\subsection{Significant Differences among the Enhanced Butter Cake}

To find out the significant difference among the four treatments sensory acceptability of the butter cake enhanced with moringa powder was conducted using the nine point hedonic scale test. From the numerical computation was revealed the most acceptable treatment. The results were tested for significant difference using the analysis of variance. The data were treated further using One-way Analysis of Variance and Two-way Analysis of Variance to determine the significant mean difference between the treatments. The one-way analysis of variance was used to test the measure of significant effects of one factor while the two-way variance tested in order to measure the effects of two factors simultaneously and whether there is an interaction between the two parameters. In one-way analysis of variance it appeared that there are no significant differences among treatments in all sensory attributes. This means that the null hypothesis is accepted which stated that there are no significant mean differences in all sensory attributes of butter cakes enhanced with moringa powder as to color, taste, flavor and texture. However in the two-way analysis of variance revealed that the sum of squares has gone from 1132.25 to the lower value of 597.52. This means that there was slight significance difference occurred between treatments. Again for example the color is affected by the amount of moringa powder present in each treatment, the more moringa powder added to the cake mixture the darker the color it becomes and the taste of malunggay is more pleasant. However the products' acceptability levels of all treatments are accepted but the more amount of moringa powder the lesser the ratings or acceptability levels given by the panelists.

\subsection{Nutritional and Economic Values of Butter Cake Enhanced with Moringa Powder}

Butter cake enhanced with moringa powder is such nutritionally rich not only from the butter cake itself but likewise enriched by the addition of moringa powder. It is now the trends that this kind of vegetable is used to be incorporated in many recipes as shown in the print and television advertisements and news broadcast. This is to capture the children consumers since mostly the adults appreciate more the moringa in take through ordinary way of preparing. But the recent utilization of moringa is not limited to one preparation but multi-purposes. In the proximate analysis it was revealed that 100 gram per slice of butter cake enhanced with moringa powder contained $13.4 \mathrm{~g}$ fat, $5.9 \mathrm{~g}$ protein and $35.2 \mathrm{~g}$ carbohydrates and 450IU of Vitamin A which means that it can supply about one eight (1/8) of the body needs. For Vitamin A, men need 3,000IU and the women need 2,333IU. This means that a slice of the butter cake enhanced with moringa can considerable meet portions of the Vitamin A daily requirements. The problem of parents to let their children eat malunggay vegetable or moringa for nutritional purposes is now partly solved by using the moringa powder to enrich recipes like the cake. Moringa is known to be a miracle vegetable plant which is used globally in many aspects such as medicinal or therapeutic, food and nutritional values. The economic value of the product is computed at Php64.95 total material cost per cake with twelve slices and sold at ten (ten) pesos per slice. This is set at eight (8) cakes per week to bake twice a week, which is to yield four (4) cakes per baking. In setting the selling price aside from the material cost, overhead, mark up and other related expenses were considered. As per computation for sixteen weeks excluding the testing weeks would come up 
with a total expenses of Php11,504.00 and could yield a gross income of php15,360.00 for one semester activity. This recorded a total of Php 3,856.00 net profit less the water, light and space rentals which were allocated as part of the expenditures monthly. Since they are students and they are paying for their tuition fees, these fees shall go to the management fund allocation which is the shop. Cost and return analysis of butter cake enhanced with moringa powder showed a return of investment (ROI) of Php.34.

The data were gathered and interpreted using hedonic test with nine scales 1 as the extremely dislike and 9 as the extremely like scale. Most acceptable formulation was determined by using the arithmetic mean. The same formulated recipe was also prepared for the proximate analysis to come up with the nutritional value of the product. The significant differences among the four formulated recipes were determined using the ANOVA formula.

Hedonic rating scale test was given to determine the degree of liking or disliking of the experienced panelists, who are composed of professors major in food technology and home economics and the consumer panelists, who are BSED and BEED students that were randomly selected to test the product. The panelists evaluated the product they indicated the degree of liking of the butter cake enhance with malunggay. The degree of liking was categorized in a hedonic rating scale of 9 points, which is the highest scale with a hedonic interpretation of like extremely, and 1 is the lowest scale with a hedonic interpretation of dislike extremely. The sensory qualities were indicated in the score sheet was given to every panelists during the sensory evaluation was conducted.

The data reflected in Table 1 is the color acceptability attribute preferred by the experienced panelists. It was observed that the hedonic mean scores of T1 (7.13), and T2 (6.93) had an interpretation of like moderately, T3 (7.6) like very much and T4 (5.93) fall to like slightly. This implies that the experienced panelists liked the product which the color is medium dark olive green which is presented in table 2.

Table 1: Hedonic Mean Acceptability Score for Color by the Experienced Panelists

\begin{tabular}{|l|l|l|l|l|l|l|}
\hline \multirow{3}{*}{ Treatment } & \multicolumn{3}{l}{ Professor Panelists } & \multicolumn{2}{l|}{ Treatment } & \multirow{2}{*}{ Total } \\
\cline { 2 - 5 } & \multicolumn{2}{|l|}{ Replication } & & & \multirow{2}{*}{ Mean } \\
\cline { 2 - 6 } & 1 & 2 & 3 & & \\
\hline 1 & 6 & 8.2 & 7.2 & 21.4 & 7.13 & Like Moderately \\
\hline 2 & 6.2 & 7.4 & 7.2 & 20.8 & 6.93 & Like Moderately \\
\hline 3 & 7.6 & 7 & 8.2 & 22.8 & 7.6 & Like Very Much \\
\hline 4 & 5 & 5.8 & 7 & 17.8 & 5.93 & Like Slightly \\
\hline Grand Total & 24.8 & 28.4 & 29.6 & 82.8 & 27.6 & \\
\hline & Student Panelists & & & \\
\hline 1 & 8.04 & 7.64 & 7.48 & 23.16 & 7.72 & Like Very Much \\
\hline 2 & 7.92 & 7.88 & 7.64 & 23.44 & 7.81 & Like Very Much \\
\hline 3 & 7.96 & 7.48 & 7.36 & 22.8 & 7.60 & Like Very Much \\
\hline 4 & 7.52 & 7.4 & 7.44 & 22.36 & 7.45 & Like Very Much \\
\hline Total & 31.44 & 30.4 & 29.92 & 91.76 & 30.58 & \\
\hline Grand Total & 56.24 & 58.8 & 59.52 & 174.56 & 58.18 & \\
\hline
\end{tabular}


Legend: 8.12-9.00 like extremely; 7.23-8.11 like very much; 6.34-7.22 like moderately; 5.456.33 like slightly; 4.56-5.44 neither like nor dislike; 3.67-4.55 dislike slightly; 2.78-3.66 dislike moderately; 1.89-2.77 dislike very much; 1.00-1.88 dislike extremely.

The enhanced butter cake with the most quantity of moringa is rated the least. Meaning, there is certain level of moringa powder to keep the product acceptable as revealed by the professor panelists.

For the student panelists, the color acceptability falls on the same categorical descriptive level in all treatments that is like very much. For them no level of moringa powder affects their color choice, anyway, all treatments are of the same hue but only of different values. It may be considered that the student panelists are all freshmen; they are very gourmand and like to eat anything that may answer their hunger and fulfill their satiety.

\subsection{Taste Attributes}

Another sensory quality measured in this study is the taste acceptability referring to one of the four types: sweet, sour, bitter and salty. In the market language, taste and flavor are synonyms. In the past, there were many products lunched out in the market without submitting for taste acceptability which resulted to product rejection by the consumers. The trend nowadays is product sampling as part of the business strategies for product marketing. Product sampling is popular in many advertisements either in print ads and television commercial ads because the taste of the pudding is in the tasting. As can be seen table 3 the professors group of panelists rated $\mathrm{T} 2$ got the highest rating scale of like very much. $\mathrm{T} 1$ and $\mathrm{T} 3$ recorded the same descriptive rating with like moderately group of evaluator with neither like nor dislike. As to taste acceptability of the product treatment with 2.5 moringa powder was the most acceptable them. It implies that tactilability threshold level of the professors' panelists limit its moringa level of addition at 2.5 tablespoons on the butter cake. In terms of taste acceptability therefore, certain level of moringa powder added to the butter cake needs to be observed because it can alter its acceptability as shown in the results of the professor panelist responses. The students' evaluation results showed that the first three treatments were rated categorically with like very much description. The fourth treatment got the least descriptive rating with like moderately. It can be noted that the students from College of Education leading to the degree of Bachelor in Secondary Education major in Business Technology are all in first year level. Young and voracious as they are may not be very critical in their sensory analysis while the professors pointed out treatment 2 as different from the rest. This means that butter cake enhanced with moringa powder if sold in freshmen level is very much acceptable in any quantity of mixture. Professors panelists are very critical as they are experienced food testers.

Table 2: Hedonic Mean Acceptability Score for Taste by the Experienced Panelists

\begin{tabular}{|c|c|c|c|c|c|c|}
\hline \multirow{3}{*}{ Treatment } & \multirow{2}{*}{\multicolumn{3}{|c|}{$\begin{array}{l}\text { Professor Panelists } \\
\text { Replication } \\
\end{array}$}} & \multicolumn{2}{|c|}{ Treatment } & \multirow{3}{*}{ Hedonic Interpretation } \\
\hline & & & & \multirow[t]{2}{*}{ Total } & \multirow[t]{2}{*}{ Mean } & \\
\hline & 1 & 2 & 3 & & & \\
\hline 1 & 5.4 & 8.2 & 7.8 & 21.4 & 7.13 & Like Moderately \\
\hline 2 & 7 & 7.8 & 8 & 22.8 & 7.6 & Like Very Much \\
\hline
\end{tabular}




\begin{tabular}{|c|c|c|c|c|c|c|}
\hline 3 & 7 & 5.8 & 6.4 & 19.2 & 6.4 & Like Moderately \\
\hline 4 & 5.8 & 4.2 & 4.6 & 14.6 & 4.86 & Neither Like or Dislike \\
\hline \multirow[t]{2}{*}{ Grand Total } & 25.2 & 26 & 26.8 & 78 & 26 & \\
\hline & \multicolumn{3}{|c|}{ Students Panelists } & & & \\
\hline 1 & 8.44 & 7.84 & 7.68 & 23.96 & 7.98 & Like Very Much \\
\hline 2 & 8.12 & 7.84 & 7.84 & 23.8 & 7.93 & Like Very Much \\
\hline 3 & 7.76 & 7.52 & 7.04 & 22.32 & 7.44 & Like Very Much \\
\hline 4 & 7.32 & 7.12 & 6.44 & 20.88 & 6.96 & Like Moderately \\
\hline Total & 31.64 & 30.32 & 29 & 90.96 & 30.32 & \\
\hline Grand Total & 56.84 & 56.32 & 55.8 & 168.96 & 56.32 & \\
\hline
\end{tabular}

\subsection{Flavor Attributes}

Flavor in the market parlance, refers to savor and essence however, in this study it means the blend of taste and aroma in the butter cake product. Table 5 shows the details of this information. In a glance from this table, revealed that treatment 1 obtained categorical descriptive ratings, like very much and treatment 2 and 3 fall on like moderately. Treatment 4 obtained categorical descriptive level of neither like or dislike. For the professors panellists, one tablespoon of moringa powder makes up the most acceptable flavor to the butter cake product but to the amount of four and a half moringa powder it alters their flavour acceptability level. The most acceptable limit therefore for moringa powder addition to butter cake is up one tablespoon but beyond this flavour acceptability is affected. However, for the students' panellists butter cakes containing one to three and a half tablespoons of moringa powder do not change their flavour acceptability since they rated treatments 1,2 and 3 with like very much verbal descriptive category range. Only treatment 4 was rated with like moderately. It can be noted again that the professors' panelists are more picky and choosy in the exercise of their expertise. Students are more concerned on the stuffing on their stomach.

Table 3: Hedonic Mean Acceptability Score for Flavor by the Experienced Panelists

\begin{tabular}{|l|l|l|l|l|l|l|}
\hline \multirow{3}{*}{ Treatment } & \multicolumn{3}{|l|}{ Professor Panelists } & \multicolumn{2}{l}{ Treatment } & \multirow{2}{*}{ Total } \\
\cline { 2 - 5 } & \multicolumn{2}{|l|}{ Replication } & Mean & \\
\cline { 2 - 5 } & 1 & 2 & 3 & & & \\
\hline 1 & 6 & 8.2 & 8 & 22.2 & 7.4 & Like Very Much \\
\hline 2 & 6.8 & 6.6 & 7.8 & 21.2 & 7.06 & Like Moderately \\
\hline 3 & 7.8 & 6 & 6 & 19.8 & 6.6 & Like Moderately \\
\hline 4 & 5.8 & 5.2 & 4.6 & 15.6 & 5.2 & Neither Like or Dislike \\
\hline Grand Total & 26.4 & 26 & 26.4 & 78.8 & 26.26 & \\
\hline & Student Panelists & & & \\
\hline 1 & 8.4 & 7.68 & 7.52 & 23.6 & 7.86 & Like Very Much \\
\hline 2 & 8.36 & 7.8 & 7.68 & 23.84 & 7.94 & Like Very Much \\
\hline 3 & 7.92 & 7.56 & 7.04 & 22.52 & 7.5 & Like Very Much \\
\hline
\end{tabular}


DOI: 10.5281/zenodo.826645

\begin{tabular}{|l|l|l|l|l|l|l|}
\hline 4 & 7.24 & 7.16 & 6.48 & 20.88 & 6.96 & Like Moderately \\
\hline Total & 31.92 & 30.2 & 28.72 & 90.84 & 30.28 & \\
\hline Grand Total & 58.32 & 56.2 & 55.12 & 169.64 & 56.54 & \\
\hline
\end{tabular}

\subsection{Texture Attributes}

Texture ability of the product in this study refers to the outer and cross section appearance of the butter cakes enhanced with moringa powder. Table 4 presents the data on this aspect.

Table 4: Acceptability for Texture

\begin{tabular}{|l|l|l|l|l|l|l|}
\hline \multirow{3}{*}{ Treatment } & \multicolumn{3}{l}{ Professor Panelists } & \multicolumn{2}{l|}{ Treatment } & \multirow{2}{*}{ Hedonic Interpretation } \\
\cline { 2 - 5 } & \multicolumn{2}{l}{ Replication } & & \multirow{2}{*}{ Mean } & \\
\cline { 2 - 6 } & 1 & 2 & 3 & & & \\
\hline 1 & 6.6 & 8.2 & 8.2 & 23 & 7.66 & Like Very Much \\
\hline 2 & 6.6 & 7.8 & 8 & 22.4 & 7.46 & Like Very Much \\
\hline 3 & 8 & 7 & 7.8 & 22.8 & 7.6 & Like Very Much \\
\hline 4 & 5.8 & 6.8 & 8 & 20.6 & 6.86 & Like Moderately \\
\hline Total & 27 & 29.8 & 32 & 88.8 & 29.6 & \\
\hline & Student Panelists & & & \\
\hline 1 & 8.28 & 7.84 & 7.92 & 24.04 & 8.01 & Like Very Much \\
\hline 2 & 8.24 & 7.68 & 8.16 & 24.08 & 8.02 & Like Very Much \\
\hline 3 & 7.8 & 7.52 & 7.84 & 23.16 & 7.72 & Like Very Much \\
\hline 4 & 7.36 & 7.8 & 7.44 & 22.6 & 7.53 & Like Very Much \\
\hline Total & 31.68 & 30.84 & 31.36 & 93.88 & 31.29 & \\
\hline Grand Total & 58.68 & 60.64 & 63.36 & 182.68 & 60.89 & \\
\hline
\end{tabular}

Looking at the data, treatments 1,2 and 3 were rated with the same descriptive category, like very much and treatment 4 was rated the least among the butter cakes enhanced with moringa powder with a descriptive hedonic categorical level of like moderately. Moringa powder variations from one to three and a half tablespoons do not alter the professors' texture acceptability but the addition of four and a half tablespoon moringa powder to the butter cake has affected the texture acceptability of the product.

The students' panelists did not see any difference in the texture appearance of all butter cakes enhanced with moringa powder as indicated in the students test results. It can be noted in all the responses of the students' panelists' hedonic rating results that these group of evaluators are not so sensitive enough to the differences on every sensory attribute being study. However, all these results need to be determined if there is significant differences in their sensory evaluation between the two group of panelists.

\section{Conclusion}

After a considerable analytical procedures conducted on the butter cake enhanced with moringa the following conclusions are here to formulated: 
The addition of moringa powder on the butter cake affected its color, taste and flavor acceptability as dictated from the results of the two-way analysis. But the texture sensory aspect being studied remained unaffected. The level or amount of moringa powder to be added to the recipe with high acceptable level should follow the experimental limit from 1 to 2.5 tablespoons moringa powder per recipe.

However, statistically it can be concluded that there is no significant difference among all treatments considering all sensory attributes being studied. Any formulation therefore, can be used although slight reduction of acceptability level can be noticed with higher amount of moringa addition to the mixture. The product is nutritionally rich and economically feasible. Based from the findings, Moringa powder can be used as additive to a butter cake. It can be integrated not only in butter cakes but also in other dishes or viand.

\section{References}

[1] Atibula, Erlinda A., A Comparative Analysis of Identified Variables in Nutrition of Naval Institute of Technology Students: Basis for a Proposed Action Plan for Nutrition Information Dissemination, May 1992

[2] Carillo, Margie B., Enhanced Cookies with Squash (Cucurbita Maxima) for Livelihood Activity, February 2004

[3] Sabondo, Erlinda P., A Comparative Study on the Effectiveness of Coco Oil and Pork Lard on Butter Cake Product: Basis for improved baking Instruction, March 1992

*Corresponding author.

E-mail address: villaganas@gmail.com 